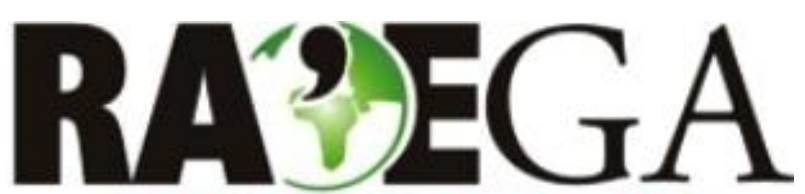

O ESPAÇO GEOGRÁFICO EM ANÁLISE

\title{
COMPATIBILIDADE VISUAL DAS REDES DE DRENAGENS DA BACIA HIDROGRÁFICA DO RIO JORDÃO (MG) EXTRAÍDAS DOS MDES SRTM E ASTER USANDO OPERADORES DE SIMPLIFICAÇÃO E SUAVIZAÇÃO
}

\section{VISUAL COMPATIBILITY OF DRAINAGE NETWORKS OF THE RIVER BASIN JORDÃO EXTRACTED FROM THE MDES SRTM E ASTER USING SIMPLIFICATION AND SMOOTHING OPERATORS}

\author{
Franciele Marques Tolentino ${ }^{1}$, Claudionor Ribeiro Silva ${ }^{2}$, Fernando Luiz de Paula \\ Santil ${ }^{3}$
}

\section{RESUMO}

A generalização cartográfica visa adaptar as feições cartográficas e as suas relações geográficas de acordo com a escala de representação do produto cartográfico. Com o advento dos computadores, os operadores permitem observar essa adaptação geométrica e semântica desse conjunto de feições segundo a função e a finalidade estabelecidas para esse produto. $O$ propósito desse trabalho foi analisar visualmente a generalização cartográfica de feições lineares extraídas do Modelo Digital de Elevação (MDE), com o uso dos operadores "simplificação" e "suavização". Foram usados os algoritmos POINT-REMOVE, para a simplificação, e o PAEK, para a suavização das redes de drenagem extraídas a partir do MDE ASTER e do MDE SRTM, respectivamente, com 30 e 90 metros de resolução espacial. Esses operadores estão implementados no software ARCGis 10.1. A área de estudo foi a bacia hidrográfica do Rio Jordão (MG). Houve controle da qualidade posicional da carta topográfica gerada em meio digital e dos MDEs e a comparação visual ocorreu com a sobreposição desses produtos. Para essa análise visual foram usados os princípios da fotointerpretação e do modelo de comunicação cartográfica. Os resultados apontam maior similitude entre os trechos lineares com a carta topográfica dessa bacia, independentemente do MDE. Outro ponto observado é que a resolução espacial e a topografia interferem na extração dessa rede de drenagem.

Palavras-chave: generalização cartográfica; feições lineares; fotointerpretação

\section{ABSTRACT}

The cartographic generalization aims at adapting the cartographic features and their geographic relations according to the scale of representation of the cartographic product. With the advent of computers, operators allow us to observe this geometric and semantic adaptation of this set of features according to the function and purpose established for this product. With the purpose of visually analyzing the cartographic generalization of linear features extracted from the Digital Elevation Model (MDE), from the application of the simplification and smoothing operators, became the focus of this work. In this case, POINT-REMOVE algorithms for simplification and PAEK were used to smooth drainage networks extracted from MDE ASTER and MDE SRTM, respectively, with 30 and 90 meters of spatial resolution. These operators are available in the ARCGis 10.1 software and the study area was the Jordan River basin (MG). There was control of the positional quality of the topographic map generated in digital media and of the MDEs and the visual comparison occurred with the overlap of these products. For this visual analysis, it was based on the principles of photointerpretation, a model of cartographic communication and the results point out a greater similarity between the linear stretches with the topographic map of this basin, independently of the MDE. On the other hand, spatial resolution and topography interfere with the extraction of this drainage network.

Key-words: Cartographic generalization; linear features; photointerpretation

\footnotetext{
1 Universidade Estadual Paulista, Presidente Prudente/SP, e-mail: francielemarques87@hotmail.com

2 Universidade Federal de Uberlândia, Uberlândia/MG, e-mail: crs.educ@gmail.com

3 Universidade Federal de Uberlândia, Uberlândia/MG, e-mail: fernando.santil@ufu.br
} 


\section{COMPATIBILIDADE VISUAL DAS REDES DE DRENAGENS DA BACIA HIDROGRÁFICA DO RIO JORDÃO (MG) EXTRAÍDAS DOS MDES SRTM E ASTER USANDO OPERADORES DE SIMPLIFICAÇÃO E SUAVIZAÇÃO}

\section{INTRODUÇÃO}

Para a representação gráfica da superfície terrestre ou de um fenômeno sempre haverá mudanças, perdas ou omissões de informações. Isto ocorre devido às restrições que são impostas pela escala de representação (DENT, 1985). Assim, o processo de adaptação de feições ou de um fenômeno a uma determinada escala é denominado generalização cartográfica (MCMASTER; SHEA, 1992; SLOCUM, 1999).

A generalização cartográfica ocorre por meio de operadores que visam adaptar geometrica e semanticamente um conjunto de feições respeitando-se a escala de representação. Tem-se por objetivo preservar as características geográficas das feições de um mapa, bem como representar suas relações espaciais e seus padrões geográficos tão fielmente quanto possível de acordo com determinada escala (MCMASTER; SHEA, 1992; TAURA et al., 2010; RAHEJA et al., 2010; NYANGWESO et al., 2016).

Dent (1985) e Slocum (1999) afirmam que a generalização cartográfica não pode ser uma simples relação de redução de escala. Este processo é um dos mais difíceis problemas colocados para o cartógrafo, principalmente por ela não ocorrer sem deformação ou deslocamento dos objetos representados. No que concerne à generalização cartográfica em ambiente digital, Nyangweso et al. (2016) argumentam que este é um processo difícil de resolver, pautado basicamente pelas exigências de flexibilidade à produção de múltiplas escalas e operações de atualização que complicam as atividades relativas à precisão, consistência e integridade dos dados. Embasado neste processo estão as aplicações com as feições lineares, por exemplo, as redes de drenagens extraídas a partir de Modelos de Elevação (MDE). Os Modelos Digitais de Elevação (MDE) representam as principais fontes de informações para a extração de feições lineares, quando comparados aos métodos tradicionais por meio de curvas de nível para atualização de produtos cartográficos, por exemplo.
O aparecimento dessas tecnologias integrado ao Sistema de Informação Geográfica (SIG), aliado à popularização dos computadores no tocante a acesso e robustez, desencadeou a evolução da produção de dados espaciais no que concerne à facilidade de extração de informações e por estar disponível para uso de todos (TOMLINSON; PETCHENIK, 1988; FAIRBAIN, 1994; BRAVO; SLUTER, 2015). Esta disponibilidade acrescenta-se ainda a internet, que permitiu a participação do usuário na produção e disseminação da informação (O'REILLY, 2007; BRAVO; SLUTER, 2015).

Esses fatos encaminham para aos chamados Sistemas de Informação Geográfica Voluntária no qual os usuários disponibilizam os conteúdos de seu interesse, contrariando a prática usual destinada aos profissionais que atuam nas atividades relacionadas ao mapeamento (GOODCHILD, 2007; BRAVO; SLUTER, 2015; NYANGWESO et al., 2016). Não se pretende avaliar um desses sistemas, como o OPENSTREETMAP, mas verificar a qualidade dos produtos espaciais gerados por meio das ferramentas anteriormente expostas. Essas são um meio para a geração de produtos cartográficos e, na maioria das vezes, se constitui na única informação disponível à extração de redes de drenagens. Avaliar a similitude desses produtos em relação à carta topográfica significa trazer à tona a relevância da compatibilidade semântica da informação que estará disponível a diferentes usuários.

No caso, os operadores de simplificação e suavização, são definidos como uma representação abstrata ou genérica descrevendo o tipo de modificação que pode ser usada quando se generaliza uma feição de interesse (NYANGWESO et al., 2016) enquanto que um algoritmo é a implementação particular desse operador, visam sob o olhar da semântica da informação cartográfica auxiliar na análise da generalização cartográfica de feições lineares (redes de drenagens) extraídas a partir de MDE. 


\section{COMPATIBILIDADE VISUAL DAS REDES DE DRENAGENS DA BACIA HIDROGRÁFICA DO RIO JORDÃO (MG) EXTRAÍDAS DOS MDES SRTM E ASTER USANDO OPERADORES DE SIMPLIFICAÇÃO E SUAVIZAÇÃO}

\section{VISÃo GERAL DA GENERALIZAÇÃo CARTOGRÁFICA DE FEIÇÕES LINEARES NO AMBIENTE DIGITAL}

Dent (1985) e Slocum (1999) apontam que a construção de um mapa requer de seu proponente uma definição das feições a serem representadas, de modo a atender os temas pertinentes ao produto, e que satisfaçam as necessidades do usuário do produto cartográfico. A escolha da escala de representação nesse processo é de grande importância, visto que esta controla o nível de detalhamento que impõe adaptação das feições cartográficas por meio de uma "simbologia". Esse processo é denominado generalização cartográfica, baseado na adaptação de feições de acordo com a escala e finalidade de um mapa (DENT, 1985). Pombo e Delazari (2014) comentam que maior será a necessidade de generalização dos objetos representados no mapa quanto maior for a razão entre elas. Esses autores complementam ainda que há dois tipos principais de generalização: semântica e a geométrica. A primeira está relacionada com a escolha inicial da informação de interesse a ser apresentada no mapa, e a segunda tem como vertente a manipulação de características gráficas de objetos representados no mapa.

A definição da escala de interesse indicará o nível de detalhamento da feição cartográfica e controlará a adaptação gráfica dela de modo a tornar legível essa representação em função do espaço disponível. Por outro lado, na representação da feição se observa dois aspectos: a relevância e característica. A relevância aponta para quais feições serão preservadas enquanto que a segunda informa a eliminação ou atenuação de características desprezíveis para acentuar essa feição (JOÃO, 2008; RAHEJA et al., 2010; POMBO; DELAZARI, 2014; NYANGWESO et al., 2016).

McMaster e Shea (1992) indicam que no meio digital a generalização ocorrerá a partir da manipulação do arquivo da representação digital de feições cartográficas enquanto que na forma convencional essa manipulação se dará pelos pontos, linhas e áreas. Sendo assim, esses autores propõe um conceito de generalização aplicado à cartografia digital: "a generalização digital pode ser definida como o processo de derivar um conjunto de dados cartográficos simbólica ou digitalmente codificada pela aplicação de transformações espaciais e de atributos a uma fonte de dados" (MCMASTER; SHEA, 1995, p. 12).

A generalização cartográfica foi decomposta por Mcmaster e Shea (1992) em três áreas operacionais: consideração dos objetivos filosóficos, que sugerem o porquê de se generalizar; avaliação das condições cartométricas, que indicam quando generalizar, e a seleção das transformações espaciais e dos atributos apropriados, que fornecem as técnicas sobre como generalizar.

Os objetivos filosóficos incluem a adesão aos princípios cartográficos e intuitivos que atendam às exigências do problema de generalização e a consideração das demandas e capacidades da tecnologia computacional disponível. A avaliação cartométrica incide sob quais procedimentos de generalização serão necessários, bem como quais medidas espaciais e holísticas pelas quais esses procedimentos foram feitos e o controle de transformação das técnicas de generalização empregadas para realizar a mudança de escala. As transformações espaciais e por atributo são modificações feitas no método, nas quais os dados são categorizados estatisticamente ou retratados simbolicamente. Esses dois tipos de transformação não são necessariamente independentes e, em muitos casos, estão intrinsecamente relacionados (MCMASTER; SHEA, 1992).

Com relação à generalização de feições lineares, de acordo com Vasconcelos e De Sá (2012), o uso do operador de simplificação de linhas reduz o número de vértices considerados redundantes, mas preserva suas propriedades e posição espacial. Essa autora indica que essa redução no número de detalhes é possível porque se busca definir pontos que tragam 0 comportamento da feição, garantindo observar o 


\section{COMPATIBILIDADE VISUAL DAS REDES DE DRENAGENS DA BACIA HIDROGRÁFICA DO RIO JORDÃO (MG) EXTRAÍDAS DOS MDES SRTM E ASTER USANDO OPERADORES DE SIMPLIFICAÇÃO E SUAVIZAÇÃO}

fenômeno independentemente da escala, e, por outro lado, se exija menor "esforço" computacionalmente para efeito de armazenamento. Este operador traz benefícios práticos que incluem: redução no tempo de traçado, aumento da nitidez da linha, armazenamento reduzido e conversão mais rápida de vetor para raster (MCMASTER; SHEA, 1992).

O operador de suavização age sobre uma linha, realocando ou deslocando a posição dos seus vértices, de modo a reduzir sinuosidades impostas pelo operador de simplificação. Essencialmente, esse operador busca captar apenas as tendências mais significativas da linha e produzir uma feição esteticamente mais agradável, tornando-a mais suave (DOUGLAS; PEUCKER, 1973; MCMASTER; SHEA, 1992).

Por outro lado, João (2008) afirma que o aumento do uso dos SIGs trouxe profundas preocupações sobre as fontes de erros em mapas, incluindo aqueles causados pelo processo de generalização cartográfica. Um dos erros mais comuns na generalização cartográfica é a decisão quanto ao nível de detalhamento ou quantidade de informações contidas em um mapa, visto que a distinção incorreta daquilo que é essencial em um mapa pode prejudicar tanto a clareza e apresentação quanto à precisão da informação (ROSSETE; MENEZES, 2014). Porém, para que o processo de generalização implique em produtos de boa qualidade, avaliação de dados espaciais vai além da questão da precisão posicional, uma vez que também, deve-se preocupar com a coerência lógica, exatidão, integridade e precisão temporal dos dados. Dessa forma, os erros que podem afetar a precisão posicional são: erros de generalização não associados com a produção do mapa de origem, erro resultante da digitalização de mapa analógico e erros devido à generalização (JOÃO, 2008).

\section{METODOLOGIA}

\section{1 ÁREA DE ESTUDO}

A área de estudo desta pesquisa é compreendida pela bacia hidrográfica do rio Jordão (BHRJ), localizada quase integralmente no município de Araguari (MG). Esta bacia está limitada pelos paralelos $18^{\circ} 50^{\prime} \mathrm{S}$ e $18^{\circ} 30^{\prime} \mathrm{S}$ e pelos meridianos $47^{\circ} 50^{\prime} \mathrm{W}$ e $48^{\circ} 10^{\prime} \mathrm{W}$, conforme ilustra a Figura 1.

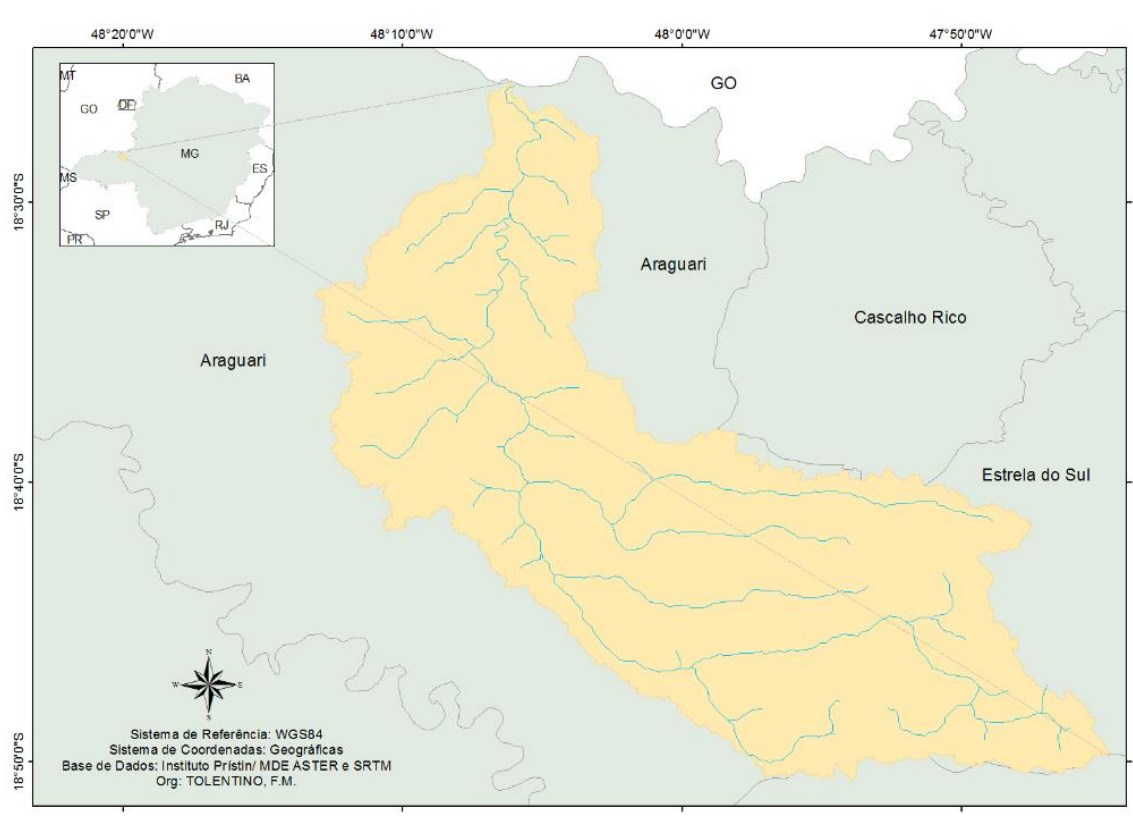

Figura 1 - Localização da bacia hidrográfica do rio Jordão. Fonte: Os autores. 


\section{COMPATIBILIDADE VISUAL DAS REDES DE DRENAGENS DA BACIA HIDROGRÁFICA DO RIO JORDÃO (MG) EXTRAÍDAS DOS MDES SRTM E ASTER USANDO OPERADORES DE SIMPLIFICAÇÃO E SUAVIZAÇÃO}

\subsection{MATERIAL}

Os MDEs SRTM (90m) e ASTER (30m) utilizados foram adquiridos junto aos sites da Empresa Brasileira de Pesquisa Agropecuária (EMBRAPA) e da empresa ASTER GDEM,

respectivamente. A Figura 2 ilustra os materiais obtidos junto aos sites mencionados.

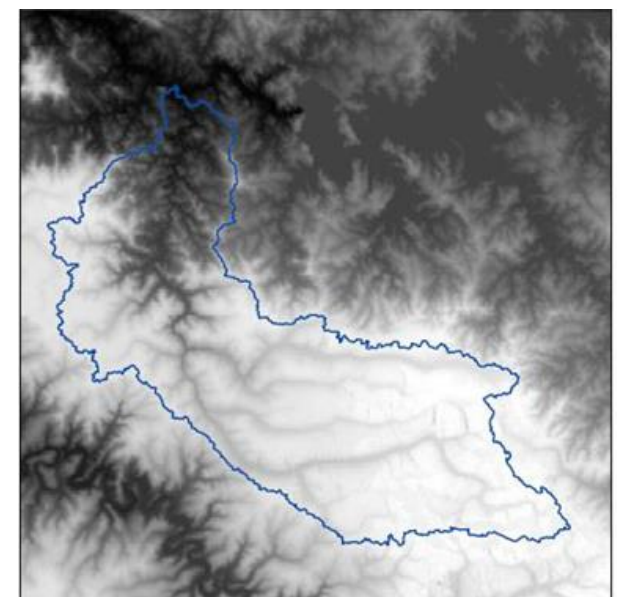

( a )

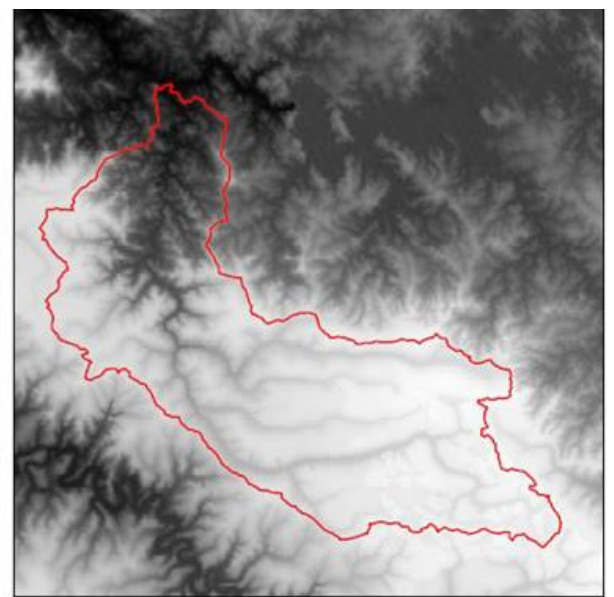

( b )

Figura 2 - Limites da bacia hidrográfica usando os MDEs ASTER (a) e SRTM (b). Fonte: Os autores.

O processo de extração automática de redes de drenagens foi realizado no software ArcGIS 10.1 usando a ferramenta Hydrology. Da mesma forma, a aplicação dos algoritmos de generalização se valeu desse software com o uso da ferramenta Generalization. Não menos importante, vale salientar que esse software está disponível no laboratório de Sistema de Informação Geográfica (SIGEO) do curso de engenharia de agrimensura e cartográfica da Universidade Federal de Uberlândia.

As cartas topográficas SE-22-Z-B-III, SE22-Z-B-VI, SE-23-Y-A-IV relativas à bacia hidrográfica do rio Jordão, na escala de 1:100.000, foram obtidas no site da Diretoria de Serviço Geográfico do Exército (DSG) e serviram para análise visual da informação cartográfica.

\subsection{VETORIZAÇÃO E CONTROLE DE QUALIDADE POSICIONAL DAS CARTAS TOPOGRÁFICAS}

A vetorização da rede de drenagem foi realizada por meio do software ArcGIS 10.1 no qual foram vetorizados os cursos d'água (redes de drenagens) da bacia hidrográfica do rio Jordão usando a ferramenta "Editor - Start Editing".
Porém, antes desse processo, foi necessário realizar o mosaico das cartas topográficas. Esta etapa foi realizada no mesmo software, usando a ferramenta Mosaic To New Raster. Deve-se mencionar que a área da bacia hidrográfica, como indicado pela Figura 1, encontra-se no mesmo fuso.

Inicialmente, foram definidos os parâmetros para edição de linhas (Tabela 1), tais como: escala de visualização, sendo esta definida de modo que fosse possível vetorizar de forma precisa os pontos centrais da feição; o modo de vetorização escolhido foi o "Passo a Passo", que permite criar passo a passo cada um dos pontos que formarão as feições lineares durante o processo de vetorização em tela; a topologia foi mantida de forma automática para que as edições topológicas sejam de responsabilidade do software; o parâmetro tolerância estabelece em qual espaço o software deve reconhecer "nós", quanto mais alto o valor, mais tolerante ele será ao juntar linhas. Destaca-se que houve preocupação em manter a escala de visualização constante, de forma a não interferir na análise visual e definição da rede de drenagem. 
SANTIL,F.L., TOLENTINO,F.M., SILVA,C.R.

\section{COMPATIBILIDADE VISUAL DAS REDES DE DRENAGENS DA BACIA HIDROGRÁFICA DO RIO JORDÃO (MG) EXTRAÍDAS DOS MDES SRTM E ASTER USANDO OPERADORES DE \\ SIMPLIFICAÇÃO E SUAVIZAÇÃO}

Tabela 1 - Valores para edição topológica.

\begin{tabular}{cc}
\hline Parâmetro & Valor \\
\hline Escala de Visualização (em tela) & $1: 3304$ \\
\hline Modo & Passo a Passo \\
Topologia & Automática \\
Tolerância & 0.1 \\
\hline
\end{tabular}

Fonte: Os autores

A verificação do produto obtido usando a vetorização manual da rede de drenagem foi obtida por intermédio do software GEOPEC, estando este de acordo com o PEC (Decreto - lei $\mathrm{n}^{\circ} 89.817$ de 20 de junho de 1984). Foram utilizados noventa pontos de controle extraídos das cartas topográficas e de suas respectivas coordenadas no produto da vetorização. Ainda foi analisada a exatidão e tendência da vetorização sendo esta última, de acordo com Santos (2010), necessária para verificar a existência de erros sistemáticos, bem como para inferir a acurácia do produto espacial. Estas análises foram realizadas por intermédio do teste de hipótese de Student (Teste t), ao nível 90\% de confiança. Para aplicação do teste foram verificadas as seguintes hipóteses: a hipótese de nulidade (HO) assumindo que os pontos não são tendenciosos e a hipótese alternativa (H1) assume que os pontos são tendenciosos.

A análise da acurácia posicional também está baseada no método de Qui-Quadrado. Segundo Santos (2010), nesse método é testado se a variância dos resíduos é estatisticamente igual à variância estabelecida pelo Decreto-Lei no 89.817/84, compatível com os valores do Erro Padrão (EP) para cada classe A+, A, B ou C.

\subsection{OPERADORES DE GENERALIZAÇÃO CARTOGRÁFICA}

O algoritmo Douglas-Peucker (simplificação) é um algoritmo recursivo e está baseado na busca de pontos críticos, assim processa o intervalo de pontos contido entre um ponto inicial (chamado de âncora) e um ponto final (chamado de flutuante). Estes dois pontos definem o segmento de uma reta. Inicialmente é determinada uma tolerância $t$, de tal forma que após traçado uma reta entre os pontos de âncora e flutuante, os pontos intermediários que possuírem uma distância $\mathrm{x}<\mathrm{t}$ serão eliminados. Em seguida, os pontos intermediários ao longo da linha curva são examinados para encontrar qual ponto tem a maior distância perpendicular entre ele e a reta definida pela âncora e o ponto flutuante. Se esta distância for inferior a distância $t$, então o segmento reto é considerado adequado para representar toda a linha. No caso em que a condição não é cumprida, o ponto mais distante torna-se o novo ponto flutuante. O ciclo é repetido, o ponto flutuante avança em direção à âncora. Quando a exigência de distância máxima é satisfeita, a âncora é movida para o flutuador e o último ponto da linha é definido como o novo ponto flutuante. Os pontos que foram atribuídos como pontos de ancoragem compreendem a linha generalizada (DOUGLAS; PEUCKER, 1973).

Uma adaptação do algoritmo DouglasPeucker, denominado POINT - REMOVE é um dos algoritmos facilmente encontrados em SIGs. Esse algoritmo atua de forma a remover vértices redundantes, assim a angularidade da feição generalizada tende a aumentar significativamente à medida que há aumento da tolerância, consequentemente a feição pode não ser esteticamente agradável (Figura 3). 


\section{COMPATIBILIDADE VISUAL DAS REDES DE DRENAGENS DA BACIA HIDROGRÁFICA DO RIO JORDÃO (MG) EXTRAÍDAS DOS MDES SRTM E ASTER USANDO OPERADORES DE SIMPLIFICAÇÃO E SUAVIZAÇÃO}

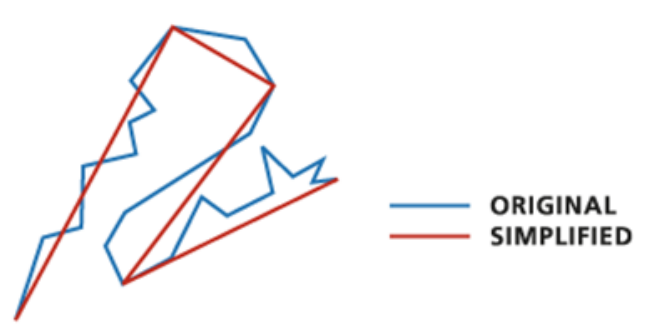

Figura 3 - Simplificação aplicada à feição linear usando o algoritmo point-remove. Fonte: ESRI - ARCGIS.

O operador suavização age sobre uma linha, realocando ou deslocando a posição dos seus vértices, de modo a reduzir sinuosidades impostas pelo operador simplificação. Essencialmente, esse operador busca captar apenas as tendências mais significativas da linha e produzir uma feição esteticamente mais agradável, tornando-a mais suave (MCMASTER; SHEA, 1992). Esse operador se mostra essencial quando se observa que as linhas geradas de modo automático, tais como as redes de drenagem e as curvas de nível, tendem a apresentar aparência angulosa. Esse operador busca uma "representação estética mais agradável", que é balizada pela ideia da "boa forma" trazida pela teoria da Gestalt (DENT, 1985; SANTIL, 2008). Assim, é possível afirmar que a suavização é um operador complementar a

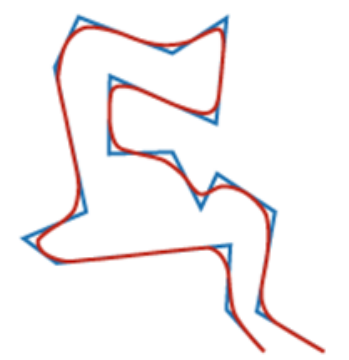

simplificação, visto que melhora a aparência angulosa causada por este.

Um dos operadores encontrados nos SIGs é o PAEK (Polynomial Approximation with Exponential Kernel). Este tem por objetivo suavizar linhas com base em uma tolerância, que especifica o comprimento do movimento do trajeto dos pontos ao longo da feição original. Assim, quanto maior a distância entre os pontos, mais suavizadas serão as linhas resultantes. 0 PAEK suaviza feições a partir da média da distância entre as coordenadas de todos os pontos da linha original. Contudo, a feição suavizada pode não possuir necessariamente todos os vértices da feição original, porém tende a manter os pontos das extremidades (VASCONCELOS; DE SÁ, 2012). A Figura 4 ilustra ação desse operador na feição linear.

Figura 4 - Suavização aplicada à feição linear usando o algoritmo point-remove. Fonte: ESRI - ARCGIS.

\subsection{DELIMITAÇÃO AUTOMÁTICA DAS REDES DE DRENAGEM}

Os Sistemas de Informação Geográfica (SIGs) tornam-se parte integrante no processo de extração de feições lineares, uma vez que estes possibilitam uma série de operações automáticas sobre os dados espaciais. Para a extração automatizada de feições lineares, como redes de drenagens por meio de MDEs, utilizaram-se algoritmos computacionais que são influenciados especialmente pela topografia do terreno da área analisada (FERNANDEZ et al., 2011). O algoritmo implementado no software ArcGIS para extração de rede de drenagens analisa as variações de altitude do relevo para determinação da direção 


\section{COMPATIBILIDADE VISUAL DAS REDES DE DRENAGENS DA BACIA HIDROGRÁFICA DO RIO JORDÃO (MG) EXTRAÍDAS DOS MDES SRTM E ASTER USANDO OPERADORES DE SIMPLIFICAÇÃO E SUAVIZAÇÃO}

do fluxo da bacia hidrográfica e de pontos de acumulação.

Segundo Bosquila et al. (2013), o método empregado no software ArcGIS para extração de redes de drenagens é o D8 (Método das Oito Possíveis Direções de Fluxo), no qual a direção do fluxo de cada pixel do MDE é atribuída de um pixel para um de seus oito vizinhos com base na diferença de cota ponderada pela distância entre eles. A delimitação da rede de drenagem utiliza o resultado dos pontos de acumulação e um limiar estabelecido pelo usuário. Assim, todo pixel que apresentar um valor acumulado maior do que o limiar apresentado pelo usuário será transformado para o valor 1 (um) do novo mapa, caso contrário, será atribuído o valor 0 (zero), gerando a rede de drenagem.

Nesse estudo, a delimitação automática da rede de drenagem foi desenvolvida por meio do software ArcGIS 10.1, seguindo os seguintes procedimentos: aplicação do comando "fill sinks", que elimina as áreas sem informação que ocasionam retenção do fluxo; cálculo da direção de fluxo ("flow direction"), que define as direções de fluxo que escoam as águas na bacia hidrográfica; obtenção da matriz com acumulação de fluxos ("flow accumulation"), que evidencia os valores de concentração de fluxo acumulado para cada célula do MDE.

Para a definição da rede de drenagem utilizou-se a ferramenta "Stream Order". Esta tem por finalidade extrair de modo hierárquico os canais de drenagem. Contudo, é gerado um arquivo com um alto número de ordem de canais, que diferem da realidade da bacia hidrográfica. O método de hierarquização da rede de drenagem adotado para esse procedimento é o proposto por Strahler (1957).

Visto que a ferramenta "Stream Order" gera uma rede de drenagem que não reflete a realidade da bacia hidrográfica, se faz necessário realizar o refinamento da mesma. Por meio do comando "Con", que limita a hidrografia às drenagens de maior acumulação de água, utilizou-se a expressão "Value" >= 4 para o MDE SRTM e "Value" >= 5 para o MDE ASTER, com a finalidade de eliminar as ordens de drenagem menores do que esses limiares. Os limiares foram definidos usando a análise visual, buscando definir qual seria o mais fidedigno à rede de drenagem da área de estudo, uma vez que limiares diferentes geram respostas de detalhamento diferentes. Por fim, após o refinamento da rede de drenagem, a rede de canais que está na forma de imagem é transformada para a forma vetorial por meio da opção "Stream to Feature".

\subsection{APLICAÇÃO DOS ALGORITMOS DE GENERALIZAÇÃO CARTOGRÁFICA}

$A$ análise de similaridades entre as redes de drenagem extraídas manualmente e de forma automática se deu a partir de amostras dos principais trechos da drenagem que compõem a bacia hidrográfica do rio Jordão (Figura 5). 


\section{COMPATIBILIDADE VISUAL DAS REDES DE DRENAGENS DA BACIA HIDROGRÁFICA DO RIO JORDÃO (MG) EXTRAÍDAS DOS MDES SRTM E ASTER USANDO OPERADORES DE SIMPLIFICAÇÃO E SUAVIZAÇÃO}
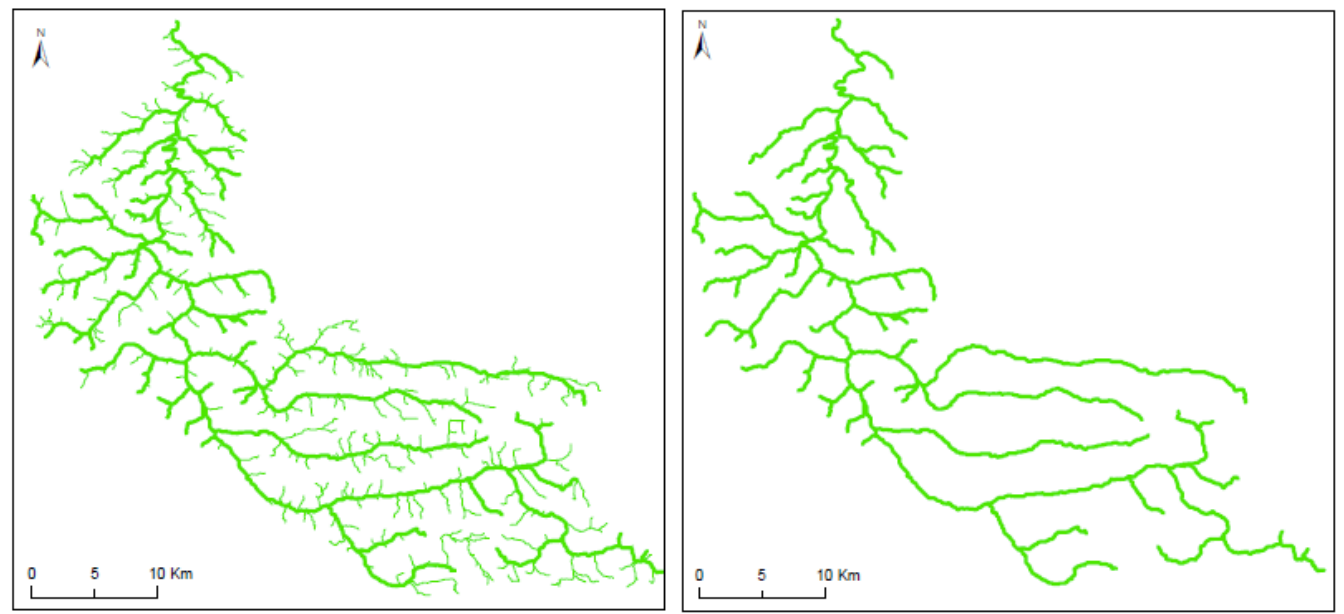

Figura 5 - Exemplo da amostra original (a) e a sua modificada (b) utilizada no MDE ASTER. Fonte: Os autores.

Em seguida, a aplicação e análise dos algoritmos de generalização cartográfica, foram feitas sobre as redes de drenagem extraídas de forma automática. Nesta etapa os algoritmos de simplificação e suavização foram aplicados. Porém, é preciso estabelecer uma tolerância que deve se relacionar com a escala em que se pretende reduzir o mapa. Assim sendo, foi definida a seguinte tolerância: o fator de acuidade visual $(0,2 \mathrm{~mm})$ multiplicado pela escala de redução. Esta proposta segue o tamanho mínimo observado a uma distância de $30 \mathrm{~cm}$, conforme se nota em Santil (2008) e Taura et al. (2010).

Desta forma, foi analisado como estes algoritmos se adaptam às feições da rede de drenagem concomitantemente com mudanças de escala. Sendo feitas as seguintes reduções: (a) para o MDE ASTER foram verificadas as escalas 1:250.000 e, sequencialmente, a escala 1:500.000 e (b) para o MDE SRTM verificou-se somente a escala 1:500.000, devido as limitações de sua resolução espacial. Para a execução dos procedimentos descritos anteriormente, utilizouse a ferramenta Cartography Tools > Generalization, disponível no software ArcGIS 10.1 .

\subsection{PROCEDIMENTOS PARA ANÁLISE VISUAL DA REDE DE DRENAGEM}

A proposta desta etapa consiste em identificar os objetos e dar significado a eles. Para isso, os procedimentos ou etapas usadas é o mesmo adotado para fotointerpretação de imagens de satélites. Aliás, é uma prática que pode ser igualmente aplicada na análise de uma fotografia comum, por exemplo. Anderson (1982) destaca três fases (ou etapas) para análise de uma imagem: identificação (ou fotoidentificação), foto-determinação e interpretação. A primeira representa a identificação do objeto e o seu significado, que é o reconhecimento desse objeto. A fase seguinte, "o usuário desenvolve processos mentais (dedutivos ou indutivos), mesmo que a imagem revele somente uma visão parcial do objeto". Finalmente, na interpretação (ou fotointerpretação), "o usuário cria correlações entre os elementos determinados na imagem e elabora hipóteses interpretativas" (PANIZZA; FONSECA, 2011, p. 37).

Com relação ao processo de comunicação do produto cartográfico, mesmo após a generalização cartográfica, o objetivo do cartógrafo (ou geógrafo) é comunicar a informação presente no mapa de forma clara e objetiva. Para se analisar esse processo de comunicação sob a feição de interesse, a rede de drenagem, adotou-se também o modelo de Board (1977).

\section{RESULTADOS E DISCUSSÕES}

A qualidade posicional após a generalização se deu a partir da análise de relatórios gerados automaticamente pelos softwares para essa confiabilidade, visualizações 


\section{COMPATIBILIDADE VISUAL DAS REDES DE DRENAGENS DA BACIA HIDROGRÁFICA DO RIO JORDÃO (MG) EXTRAÍDAS DOS MDES SRTM E ASTER USANDO OPERADORES DE SIMPLIFICAÇÃO E SUAVIZAÇÃO}

na tela com os parâmetros de restrições de simbolização e sua análise visual, bem como o comportamento da linha nas escalas selecionadas. Além disso, avaliações cartométricas entre as variações de deslocamento e comprimento da feição linear foram também analisadas.

Análise da exatidão e tendência da vetorização, de acordo com Santos (2010), é necessária para verificar a existência de erros sistemáticos e inferir a acurácia do produto espacial. Estas análises foram realizadas por meio do teste de hipótese de Student (Teste $\mathrm{t}$ ), ao nível $90 \%$ de confiança. O teste estatístico indicou que produto é preciso e não apresenta tendência, sendo este acurado para a escala de 1:100.000. Com relação análise da acurácia posicional para a escala das cartas topográficas (1:100.000) o produto foi classificado de acordo com a classe At, conforme aponta a Tabela 2 .

Tabela 2 - Resultado da análise posicional para vetorização das cartas topográficas.

\begin{tabular}{ccccc}
\hline Classe & $\mathbf{X}^{2}$ tab & $\mathbf{X}^{\mathbf{2}} \mathbf{d i}(\mathbf{E})$ & $\mathbf{X}^{\mathbf{2}} \mathbf{d i}(\mathbf{N})$ & Resultado \\
\hline A+ & 106,2033 & 0,1907 & 0,0637 & Aprovado \\
A & & 0,0612 & 0,0205 & Aprovado \\
B & & 0,0220 & 0,0074 & Aprovado \\
C & & 0,0513 & 0,0051 & Aprovado \\
\hline
\end{tabular}

Fonte: Os autores.

A análise da comunicação da informação a partir do modelo de Board (1977) tem-se:

(a) fase de elaboração do mapa: há redução progressiva da informação até ser representada graficamente por meio de um mapa. Sabe-se que os mapas são, principalmente, representações gráficas da realidade observada (SLOCUM, 1999), na grande maioria das vezes o principal estímulo sensorial que capta as informações nele contidas é o da visão (ARNHEIM, 1976; BRAVO; SLUTER, 2015). É interessante notar que, apesar dessa representação, existe o desconhecimento por quem elaborou da confiabilidade e compatibilidade desse produto tomado como referência e o gerado por apoio do computador. Isto ocorrerá na leitura do mapa, momento em que se fará a verificação em campo dos objetos de interesse: a similitude da feição com as relações espaciais observadas no produto gerado.

(b) fase de reconhecimento e identificação: são as etapas iniciais na leitura do mapa. Nota-se nas Figuras 6 e 7 a forma linear indicado pela linha verde da geometria do objeto, bem como o comportamento dessa feição nas duas escalas de trabalho se assemelham e a sua localização há pequenas variações quando sobrepostas à linha de referência. É claro, sabe-se que essa feição com forma linear e sinuosa tem o comportamento de um rio. Nesta fase há associação entre o conhecimento existente e o indicado pela representação gráfica, gerando a decodificação do objeto. 


\section{COMPATIBILIDADE VISUAL DAS REDES DE DRENAGENS DA BACIA HIDROGRÁFICA DO RIO JORDÃO (MG) EXTRAÍDAS DOS MDES SRTM E ASTER USANDO OPERADORES DE SIMPLIFICAÇÃO E SUAVIZAÇÃO}
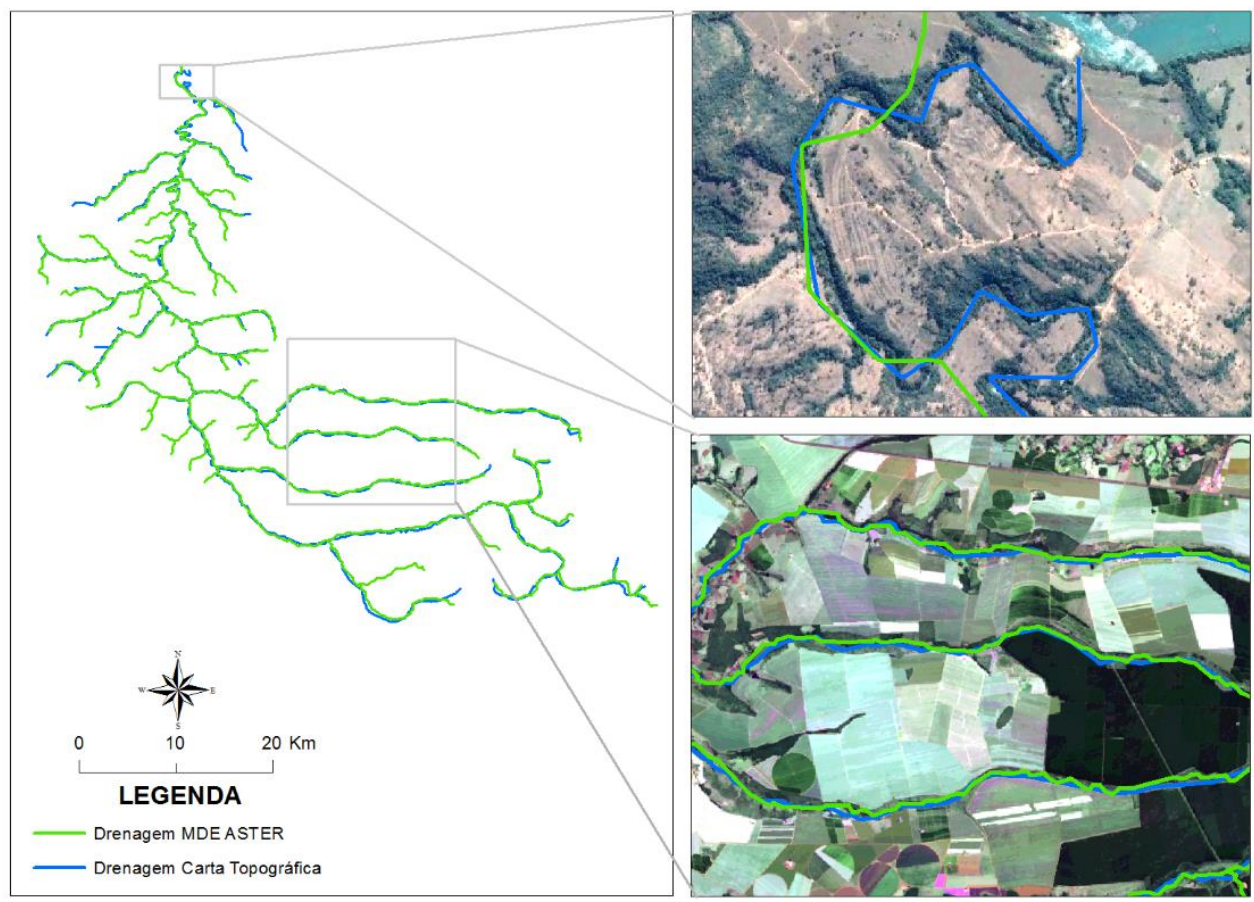

Figura 6 - Comparação visual entre carta topográfica vs MDE ASTER na escala 1:250.000. Fonte: Os autores.
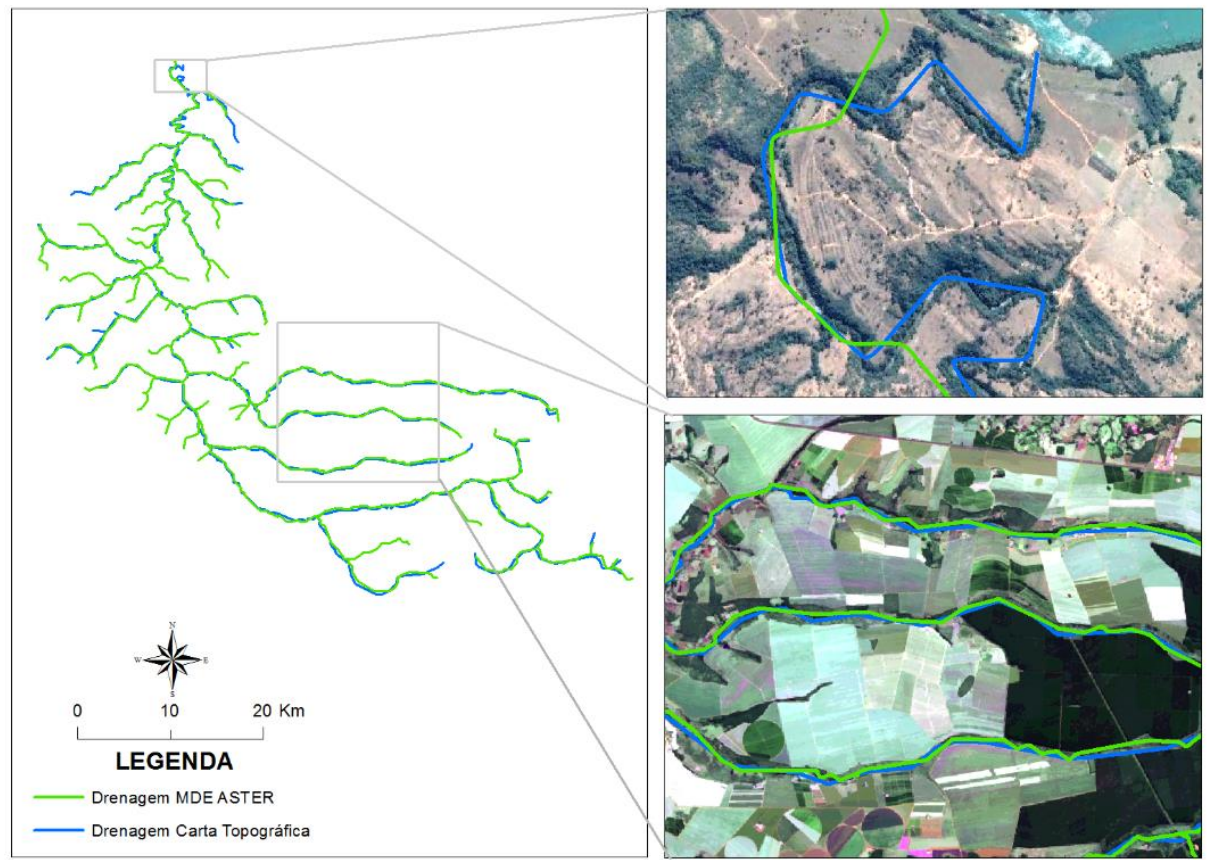

Figura 7 - Comparação visual entre carta topográfica vs MDE ASTER na escala 1:500.000. Fonte: Os autores.

Outro fator observado é a textura que aponta o menor aspecto perceptível observado em função da escala. No caso, a feição é orientada conforme as características do relevo. A informação contida nesse produto cartográfico é transferida para o campo cognitivo do usuário, porém uma determinada proporção permanece fora do seu conhecimento. Isso ocorre devido a fatores resultantes do desconhecimento do usuário com relação ao comportamento da feição, do princípio da reflectância dos alvos, entre outros. 


\section{COMPATIBILIDADE VISUAL DAS REDES DE DRENAGENS DA BACIA HIDROGRÁFICA DO RIO JORDÃO (MG) EXTRAÍDAS DOS MDES SRTM E ASTER USANDO OPERADORES DE SIMPLIFICAÇÃO E SUAVIZAÇÃO}

Por exemplo, o MDE ASTER apresenta melhor resolução espacial, verifica-se que muitas vezes este não apresenta adequabilidade para aplicação em trabalhos de média e grande escalas. Esse fato se justifica porque, mesmo após a redução da rede de drenagem extraída a partir desse MDE, para as escalas 1: 250.00 e 1: 500.000 , se notou que em muitos trechos das feições em teste a drenagem apresentou aspecto sinuoso e com grandes variações para pequenas distâncias, porém permanecendo com suas características originais. Isto se assemelha aos resultados apresentados em Taura et al. (2010) e Nyangweso et al. (2016).

Por sua vez, as maiores concordâncias, entre a rede de drenagem em teste e a de referência, após generalização cartográfica, ocorrem nas partes mais baixas e mais planas da bacia hidrográfica, e as maiores diferenças no aspecto das feições ocorrem nas áreas em que a bacia possui maior declividade tanto para a escala 1:250.000 (Figura 6) quanto para a escala
1:500.000 (Figura 7). Para facilitar essa visão, optou-se por colocar a imagem do Google Earth como fundo e sobre ela aplicou-se as redes de drenagem obtidas via carta topográfica e MDE ASTER.

Após a rede de drenagem extraída a partir do MDE SRTM ser reduzida para a escala 1 : 500.000, observou-se expressivas variações em locais onde a drenagem possui muitos meandros, quando comparada com a rede de drenagem extraída de cartas topográficas. Por outro lado, locais onde a drenagem apresenta perfil linear, verifica-se a permanência da similaridade e, consequentemente, uma adequação entre a rede de drenagem generalizada e a original (Figura 8). Esses resultados se mostram coerentes, pois locais onde a drenagem possui muitas variações há mais pontos para serem reduzidos, visto que é necessária uma quantidade maior de pontos para compor a feição, diferente do que ocorre em drenagens com perfil retilíneo.
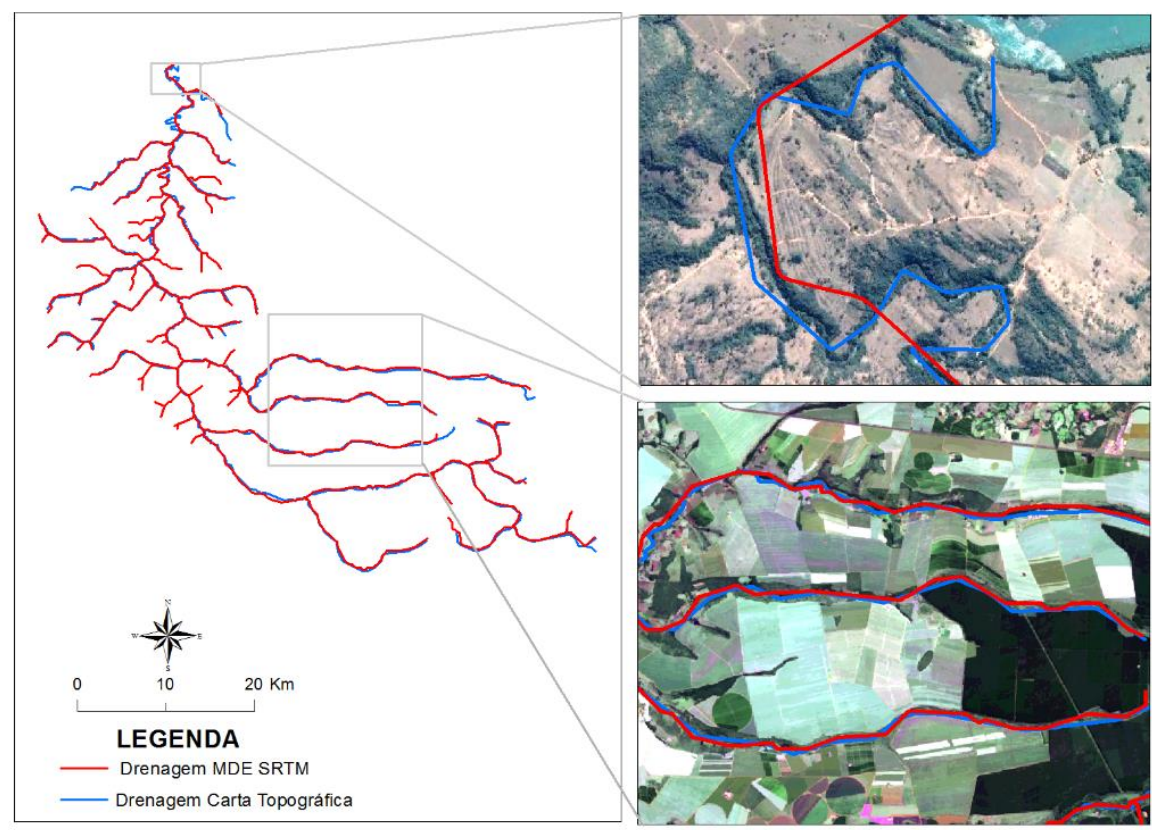

Figura 8 - Comparação visual entre carta topográfica vs MDE SRTM na escala 1:500.000. Fonte: Os autores.

(c) No processo de comunicação do mapa, seguindo-se a proposta do modelo de Board (1977) e os indicativos estabelecidos por Anderson (1982), faltam duas atividades: avaliação e a verificação. Elas ocorrem após a informação ter sido transferida do mapa para a cognição do leitor. Assim, a avaliação é o termo aplicado à busca do grau de eficácia do mapa em relação aos interesses do leitor. Como a proposta é verificar as semelhanças entre os produtos gerados e o tomado por referência pode-se mencionar que há extração das feições relativas a 


\section{COMPATIBILIDADE VISUAL DAS REDES DE DRENAGENS DA BACIA HIDROGRÁFICA DO RIO JORDÃO (MG) EXTRAÍDAS DOS MDES SRTM E ASTER USANDO OPERADORES DE SIMPLIFICAÇÃO E SUAVIZAÇÃO}

drenagem no grau compatível com a escala de análise. Entretanto, a verificação que trata do processo de avaliação do mapa no campo notouse discrepância na montante do rio Jordão para ambos os MDEs. O trabalho de campo permitiu detectar os possíveis "erros" na geração desse elemento. Verificou-se que o não comportamento esperado na nascente desse rio se deve a uma "bifurcação" decorrente da presença de áreas úmidas que ocorrem na época de cheia e o conduz a traçar uma "linha alternativa" a que de fato corresponde.

A partir da rede de drenagem extraída de forma automática é possível verificar, por meio da análise visual, que se têm melhores resultados na jusante da bacia hidrográfica. Este fato se justifica em função dos algoritmos de extração automática de redes de drenagem porque estes se baseiam, principalmente, nas variações de altitude do relevo. Quanto mais ondulado for o terreno, mais preciso o algoritmo será para reconhecer canais de drenagem. Nesse sentido, devido a melhor resolução espacial do MDE ASTER (Figura 9b), este apresentou uma rede de drenagem mais densa e com melhor traçado quando comparada com a extraída do MDE SRTM (Figura 9c). Apesar da resolução espacial do ASTER se melhor do que a do SRTM, nota-se ainda que há canais que não são evidenciados pela rede de drenagem extraída manualmente, indicando um possível de erro de comissão.

Outro ponto pertinente se relaciona aos valores adotados na Tabela 1 para visualização da rede de drenagem. Apesar da Figura 9 não apresentar essa visualização é possível observar que o tamanho mínimo apontado para feição em análise está correto, conforme nota nos resultados apresentados por Santil (2008) e Taura et al. (2010).
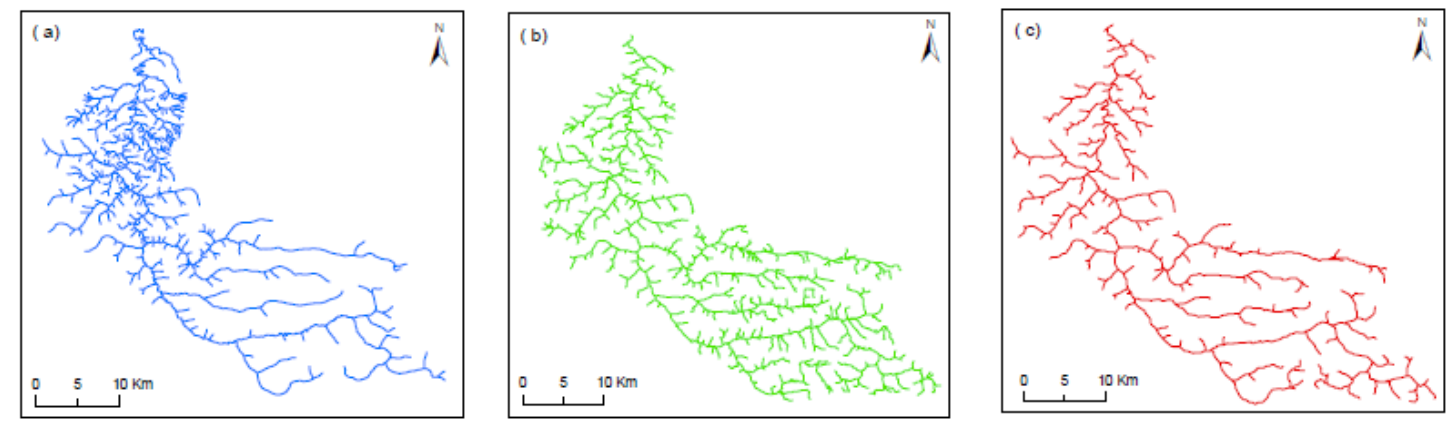

Figura 9 - Comparação visual entre carta topográfica (a), MDE ASTER (b) e MDE SRTM (c). Fonte: Os autores.

Em relação a análise cartométrica, observou-se que o ponto da foz do Rio Jordão nos MDEs ASTER e SRTM apresentou um deslocamento na sua posição, quando comparados com a extração da rede de drenagem pelo método manual, derivada de cartas topográficas. Este fato se baliza pelos algoritmos de extração automática se "orientarem" na direção de fluxo do rio concomitantemente com as variações de declividade ao longo do canal. Isto significa que os algoritmos simulam o caminho preferencial para o escoamento superficial da água, assim observou-se que o algoritmo presente no software ArcGIS 10.1 extraiu a rede de drenagem da bacia até o ponto de menor altitude, determinando-o como o ponto de foz (Tabela 3).
De acordo com Tabela 3, nota-se que apesar de se ter encontrado resultados satisfatórios para o delineamento de rede de drenagem através do MDE ASTER, este foi o que apresentou maior discrepância $(850 \mathrm{~m})$ em relação ao deslocamento do ponto da foz (Figura 10), devido a sua resolução espacial ser melhor do que o MDE SRTM e este possui maior sensibilidade em identificar diferenças topográficas do relevo. Percebe-se a importância do operador em saber avaliar a potencialidade de MDEs em contraste com a realidade do ambiente, pois, caso ele não esteja atento a tais necessidades técnicas, a execução e a qualidade dos produtos cartográficos gerados a partir desses modelos podem ser comprometidas. 


\section{COMPATIBILIDADE VISUAL DAS REDES DE DRENAGENS DA BACIA HIDROGRÁFICA DO RIO JORDÃO (MG) EXTRAÍDAS DOS MDES SRTM E ASTER USANDO OPERADORES DE SIMPLIFICAÇÃO E SUAVIZAÇÃO}

Tabela 3 - Variação da altitude dos pontos de foz.

EXTRAÇÃO DA REDE DE DRENAGEM ～ALTIDUDE DO PONTO DE FOZ (M)

$$
\text { CARTA TOPOGRÁFICA }
$$

MDE ASTER

MDE SRTM
532

526

536

Fonte: Os autores.

Apesar dos MDEs originalmente já possuírem sistema de referência, estes apresentam deformações em sua qualidade geométrica que precisam ser corrigidas como propósito de avaliar a sua acuracidade. 0 referenciamento dos MDEs seguiu-se a mesma proposta descrita anteriormente para 0 referenciamento das cartas topográficas, porém tendo estas como base para o processo. Portanto, definidos os pontos de controle e posicionando-os com exatidão sobre as cartas topográficas, foi possível a indicação de um RMS baixo, compatível com a escala utilizada dos MDEs (Tabela 4).
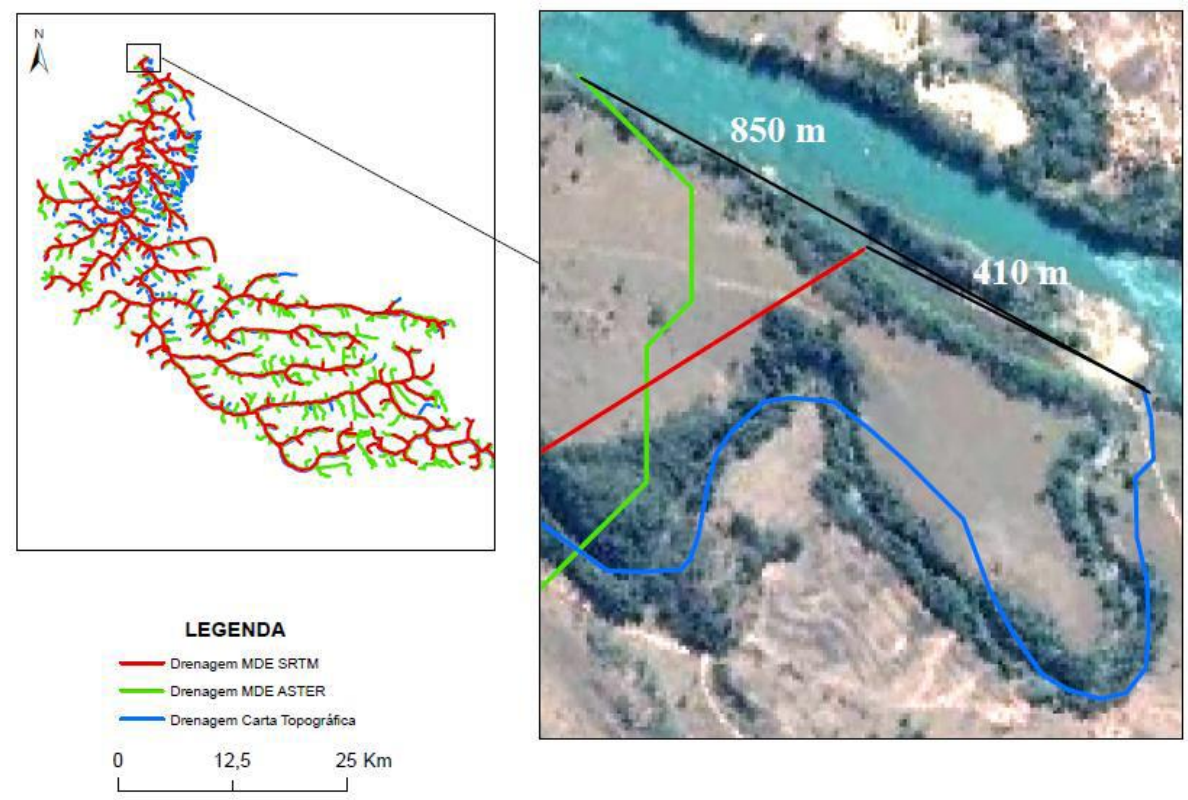

Figura 10 - Deslocamento da nascente do rio Jordão (MG). Fonte: Os autores.

Tabela 4 - Valor do erro médio quadrático para os registros do MDEs.

\begin{tabular}{cc}
\hline MDE & RMS (M) \\
\hline SRTM & 204,58 \\
ASTER & 114,32 \\
\hline
\end{tabular}

Fonte: Os autores. 


\section{COMPATIBILIDADE VISUAL DAS REDES DE DRENAGENS DA BACIA HIDROGRÁFICA DO RIO JORDÃO (MG) EXTRAÍDAS DOS MDES SRTM E ASTER USANDO OPERADORES DE SIMPLIFICAÇÃO E SUAVIZAÇÃO}

\section{CONSIDERAÇÕES FINAIS}

Este trabalho se propôs a generalizar as redes de drenagens extraídas dos modelos SRTM e ASTER GDEM usando os operadores de simplificação e suavização. Nessa ordem, os operadores de simplificação e suavização de feições lineares tendem a reduzir o número de pontos de uma feição, de modo a simplificar as informações representadas em um mapa e suavizar a aparência dessa feição de modo a torna-la mais agradável esteticamente.

Para a generalização cartográfica foi necessário estabelecer uma tolerância que se relacionasse com a escala pretendida à redução do mapa, para isso foi relacionado o fator de acuidade visual com a escala de redução. Apesar dos resultados terem sido satisfatórios, recomenda-se que sejam feitos outros testes com outros valores de tolerância, visto que esta etapa ainda é muito subjetiva.

As transformações de escala 1:250.000 e, sequencialmente, 1:500.000 no MDE ASTER, além da redução para a escala de 1:500.000 no MDE SRTM, mostraram-se satisfatórias uma vez que em muitos locais foram mantidas as condições originais da rede de drenagem. Em uma análise visual, pode-se afirmar que os resultados obtidos em ambos os MDEs são similares. Contudo, destaca-se que a topografia do terreno e a resolução espacial dos MDEs são características fundamentais, para o entendimento da rede de drenagem e, consequente, para sua generalização. Mas para a avaliação de um mapa o trabalho de campo continua fundamental de forma a minimizar possíveis erros e aprimorar as técnicas existentes. No caso, aplicar os demais operadores de generalização cartográfica envolvendo situações como a variação da topografia e outras feições da carta topográfica (TAURA et al., 2010).

Com relação à análise visual (visão), no entendimento das pesquisas cognitivas em Cartografia, é um sistema de processamento da informação (SANTIL; SLUTER, 2012). Esse processo é denominado por cognição visual e Pinker (1984) afirma que ela ajuda compreender o processo de aquisição do conhecimento espacial e pode ser um talento que ajude a compreensão da inteligência humana. Como afirmam Ardilla et al. (2010), esse processo está interligado a outros processos, como os da capacidade humana de aprender ou repetir padrões de comportamento por meio da observação.

Pinker (1984) destaca que esse processo envolve, por um lado, a forma como os indivíduos raciocinam a representação visual do mundo e, por outro, o processo de relembrar ou raciocinar sobre os objetos e feições existentes. $\mathrm{O}$ segundo processo descrito por esse autor ficará como uma futura proposta de discussão, pois neste campo, de acordo com Bravo e Sluter (2015), estuda-se a categorização mental, os processos de classificação da informação, assim como, as estruturas de organização do conhecimento no qual os demais operadores poderão auxiliar nesse processo da visualização da informação espacial.

\section{REFERÊNCIAS}

ANDERSON, P. S. Fundamentos de fotointerpretação. Rio de Janeiro: SBC, 1982.

ARDILLA, A.; BERTOLUCCI, P. H.; BRAGA, L. W.; CASTRO-CALDAS, A.; JUDD, T.; KOSMIDIS, M. H.; MATUTE, E.; NITRINI, R.; OSTROSKY-SOLIS, F.; ROSSELLI, M. Illiteracy: the Neuropsychology of Cognition Without Reading. Archieves of Clinical Neuropsychology, Oxford, vol. 25, p. 689-712, 2010.

ARNHEIM, R. T. The perception of maps. The American Cartographer, Washington, vol. 3, n.1, p. 5-10, 1976.

BOARD, C. The geographer's contribuition to evaluating maps as vehicles for communicating information. In: INTERNATIONAL YEARBOOK OF CARTOGRAPHY, 17, 1977, Hamburg: ICACI, 1-4/9, p. 47-59.

BOSQUILA, R.W.D.; FIORIO, P. R.; BARROS, P. P. da S.; MARTINS, J. A.; DUARTE, S. N. Comparação entre modelos de mapeamento automático de drenagens usando SIG. In: SIMPÓSIO BRASILEIRO DE SENSORIAMENTO REMOTO, 16, 2013, Foz do Iguaçu: INPE, 13-18/04, p. 5872 - 5879.

BRAVO, J. V. M.; SLUTER, C. R. O problema da qualidade de dados espaciais na era das informações geográficas voluntárias. Boletim de Ciências Geodésicas, Curitiba, vol. 21, n. 1, p. 5673, 2015. 


\section{COMPATIBILIDADE VISUAL DAS REDES DE DRENAGENS DA BACIA HIDROGRÁFICA DO RIO JORDÃO (MG) EXTRAÍDAS DOS MDES SRTM E ASTER USANDO OPERADORES DE SIMPLIFICAÇÃO E SUAVIZAÇÃO}

DENT, B. D. Principles of thematic map design. Massachusetts: Addison-Wesley, 1985.

DOUGLAS, D. H.; PEUCKER, T. K. Algorithms for the Reduction of the Number of Points Required to Represent a Digitized Line or its Caricature. The Canadian Cartographer, Toronto, v.10, n. 2, p. 112-122, 1973.

FAIRBAIN, D. J. The frontier of cartography: mapping a changing discipline. Photogrammetric Record, Nottingham, vol.14, n. 84, p. 903-915, 1994.

FERNANDEZ, D. C. J.; VALERIANO, M. M.; ZANI, H.; FILHO ANDRADES, C. O. Algoritmos de fluxo para extração de redes de drenagem por processos automáticos a partir de modelos digitais de elevação. In: SIMPÓSIO BRASILEIRO DE SENSORIAMENTO REMOTO, 15, 2011, Curitiba: INPE, 30/4-5/5, p. $5163-4170$

GOODCHILD, M. F. Citizens as sensors: the world of Volunteered Geography. GeoJournal, Amsterdam, vol.69, p. 211-221, 2007.

JOÃO, E. M. Causes and consequences of map generalization. Bristol: Taylor \& Francis Inc., 1998.

MCMASTER, R. B.; SHEA, K.S. Generalization in digital cartography. Washington: Association of American Geographers, 1992.

NYANGWESO, D. N.; NJOROGE, T. M.; SIRIBA, D. N. Cartographic Generalization in Multi-scale Environment: case study of Lamu County, Kenya. International Journal of Science and Research, Delhi, vol. 5, p. 804-813, 2016.

O'REILLY, T. What is Web 2.0: Design patterns and business models for the next generation of software. Communications \& Strategies, Sweden, vol.1, n. 17, p. 17-37. 2007.

PANIZZA, A. C.; FONSECA, F. P. Técnicas de interpretação visual de imagens. Espaço e tempo, São Paulo, n. 30, p. 30-43, 2011.

PINKER, S. Visual Cognition: An Introduction. Cognition, Rockville Pike, vol. 18, p. 1-63, 1984.

POMBO, R. M.; DELAZARI, L. S. Automação dos operadores de agregação e deslocamento para generalização cartográfica na representação de redes sociais. Boletim de Ciências Geodésicas, Curitiba, v. 20, n. 3, p. 669-682, 2014.

RAHEJA, J. L.; KUMAR, U.; SARAVANAN, K. C. Cartogrhapic generalization (selection) for 2D map of urban area. International Journal of Computer Science \& Information Techonology, Delhi, vol. 2, n. 1, p. 69-75, 2010.

ROSSETE, A. C.; MENEZES, P.M.L. Erros comuns na cartografia temática. In: XXVI CONGRESSO BRASILEIRO DE CARTOGRAFIA, 26, 2014, Gramado: SBC/UFRGS, 03-07/08, s/n.

SANTIL, F. L. P. Análise da percepção das variáveis visuais de acordo com as leis da Gestalt para representação cartográfica. 2008. Tese (Doutorado em Ciências Geodésicas) Departamento de Geomática, Universidade Federal do Paraná, Curitiba.

SANTIL, F. L. P.; SLUTER, C. R. As pesquisas em cognição visual aplicadas à Cartografia. Revista Brasileira de Cartografia, Rio de Janeiro, vol. 64, n. 3, p. 367-376, 2012.

SLOCUM, T. A. Thematic cartography and visualization. New York: Prentice-Hall, 1999.

STRAHLER, A. N. Quantitative Analysis of Watershed Geomorphology. Transactions American Geophysical Union, Washington, v.38, $n^{\circ} 6$, p. $913-920,1957$.

TAURA, T. A.; SLUTER, C. R.; FIRKWOSKI, H. Generalização cartográfica das cartas do mapeamento urbana nas escalas 1:2000, 1:5000 e 1:10000. Boletim de Ciências Geodésicas, Curitiba, vol. 16, n. 3, p. 386-402, 2010.

TOMLINSON, R. F.; PETCHENIK, B. B. Reflections on the revolution: the transition of analogue to digital representations of space. The American Cartographer, Washington, vol. 15, n.3, p. 249261. 1988.

VASCONCELOS, T. L.; DE SÁ, L. A. C. M. Generalização Cartográfica de Feições Lineares. In: SIMPÓSIO BRASILEIRO DE CIÊNCIAS GEODÉSICAS E TECNOLOGIAS DA GEOINFORMAÇÃO, 9, 2012, Recife, 06-09/5, p. 01 -08 . 